\title{
Functional characterization of human oncoprotein gankyrin in Zebrafish
}

\author{
So Yeon $\mathrm{Kim}^{1}$, Wonhee Hur, ${ }^{1}$ \\ Jung-Eun Choi ${ }^{1}$, Daniel Kim ${ }^{1}$, \\ Jin Sang Wang', Hye-Yeon Yoon', \\ Lian-Shu Piao ${ }^{1}$ and Seung Kew Yoon ${ }^{1,3}$ \\ 1Department of Internal Medicine and \\ WHO Collaborating Center of Viral Hepatitis \\ College of Medicine \\ The Catholic University of Korea \\ Seoul 137-701, Korea \\ ${ }^{2}$ Department of Life Science \\ The Ewha Womens University \\ Seoul 158-056, Korea \\ ${ }^{3}$ Corresponding author: Tel, 82-2-590-2622; \\ Fax, 82-2-536-9559; E-mail, yoonsk@ catholic.ac.kr \\ DOI 10.3858/emm.2009.41.1.002
}

Accepted 22 September 2008

Abbreviations: BrdU, 5'-bromodeoxyuridine; hpf, hours post fertilization

\begin{abstract}
Gankyrin is an oncoprotein containing seven ankyrin repeats that is overexpressed in hepatocellular carcinoma (HCC). Gankyrin binds to Mdm2, which results in accelerated ubiquitylation via degradation of $p 53$, and it also plays an important role in cell proliferation. However, little is known about the relationships between p53 levels, cell proliferation, and gankyrin over-expression. In order to investigate the influence of gankyrin protein on p53 and Mdm2 in a zebrafish model, we injected human gankyrin (hgankyrin) containing expression vectors (pCS2-hgankyrin, pCS2hgankyrin-EGFP) into zebrafish embryos. To measure p53 and Mdm2 expression in hgankyrin-injected embryos, RT-PCR, Northern blot and in-situ hybridization and BrdU immunostaining were used. In addition, to know the effect of hgankyrin on cell proliferation in vitro, cell viability assays such as MTT, trypan blue staining and RT-PCR following transfection of hgankyrin-containing vector into HEK 293 cell line were performed. In vivo results indicated that p53 mRNA levels decreased but those of Mdm2 were not decreased in the presence of hgankyrin. These results suggest that gankyrin downregulates p53 expression and not Mdm2
\end{abstract}

expression. In the study of cell proliferation, BrdU-positive cells were predominantly increased in the head and tail regions in hgankyrin-injected zebrafish. Additional in vitro studies using trypan blue staining and MTT assay showed that gankyrin-expressing HEK 293 cells proliferated at a faster rate, indicating that gankyrin promotes cell proliferation. Our results demonstrate that hgankyrin overexpression downregulates p53 expression and promotes cell proliferation in zebrafish. Gankyrin may play an important role in tumorigenesis via its effects on $\mathrm{p} 53$ and cell proliferation.

Keywords: cell proliferation; proto-oncogene proteins c-mdm2; PSMD10 protein, human; tumor suppressor protein $\mathrm{p53}$; zebrafish

\section{Introduction}

Human oncogenic protein gankyrin is reported to be overexpressed in hepatocellular carcinoma (HCC) (Higashitsuji et al., 2000; Park et al., 2001). Gankyrin consists of 226 amino acids including seven ankyrin repeats and forms a complex with the S6b ATPase subunit of the human 26s proteasome (Krzywda et al., 2004), which interact with other proteins (Lozano, 2005). LxCxE binding motif exiting in gankyrin interacts with retinoblastoma (RB1), which promotes the progression of tumor formation through the cell cycle by causing the release of E2F. Also, gankyrin binds to CDK4 and forms the gankyrin-CDK4-Cyclin $D$ ternary complex structure, which promotes G1/S progression (Qin et al., 2005).

The up-regulation of gankyrin is related to cell cycle progression and the proliferation of hepatoma cells (Iwai et al., 2003) and normal hepatocytes (Li and Tsai, 2002). Its overexpression in cultured $\mathrm{NIH}-3 \mathrm{~T} 3$ cells results in the transformation of these cells and injection into nude mice leading to tumor formation (Krzywda et al., 2004). Recent studies focusing on the binding of gankyrin to Mdm2, has been shown to reduce the level of the p53 protein by increasing its ubiquitylation and degradation (Higashitsuji et al., 2005).

Namely, gankyrin regulates ubiquitylation of p53 through Mdm2 and is an adaptor protein that delivers ubiquitylated p53 to the 26 s proteasome (Higashitsuji et al., 2005). Gankyrin interactions with proteins, of which Mdm2 is one, lead to the 
degradation of p53 through the ubiqutination proteasome pathway and increase in cell proliferation. This phenomeon has not yet demonstrated in vivo study, hence, we sought to demonstrate these pathways using zebrafish models. Gankyrin was mapped to chromosome Xq22.3 and BLAST searches revealed that it has $35 \%$ homology with a gene in yeast (Nas6p) and $78 \%$ homology with a gene in zebrafish (proteasome 26s subunit, non-
ATPase; data not shown) suggesting that it has a crucial function in cells.

The zebrafish model system has many advantages, since zebrafish have a short life cycle, small body size and easy embryonic manipulation (Amatruda et al., 2002). Zebrafish is also an ideal vertebral tumor model system as it has a cell cycle, tumor suppressor and oncogenes in common with those found in humans. Accordingly, new mole-

A

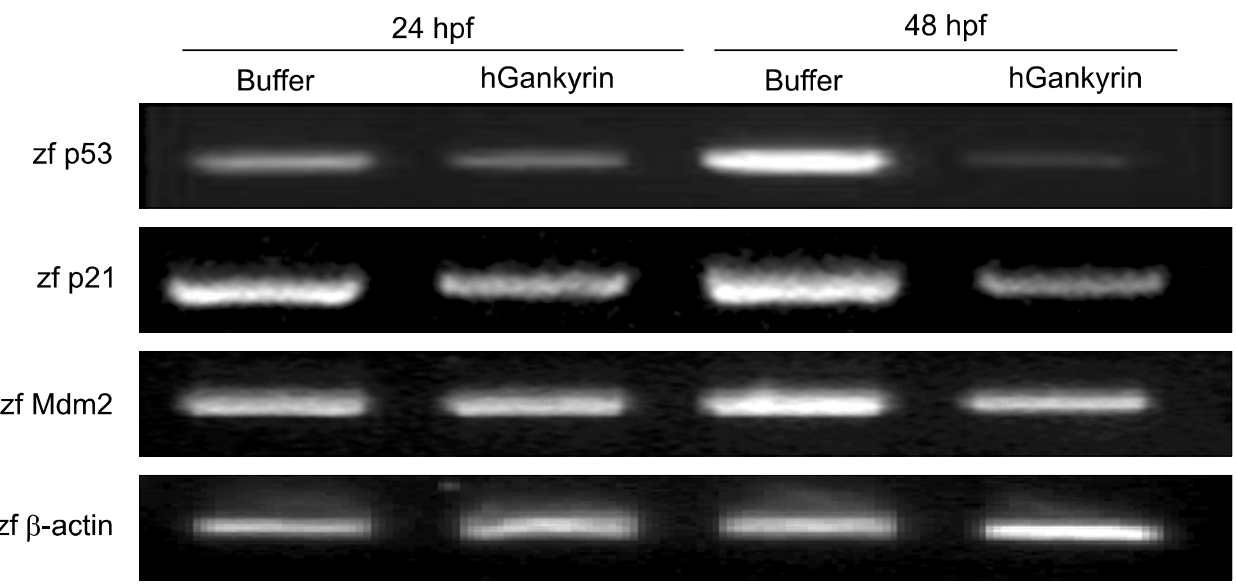

B

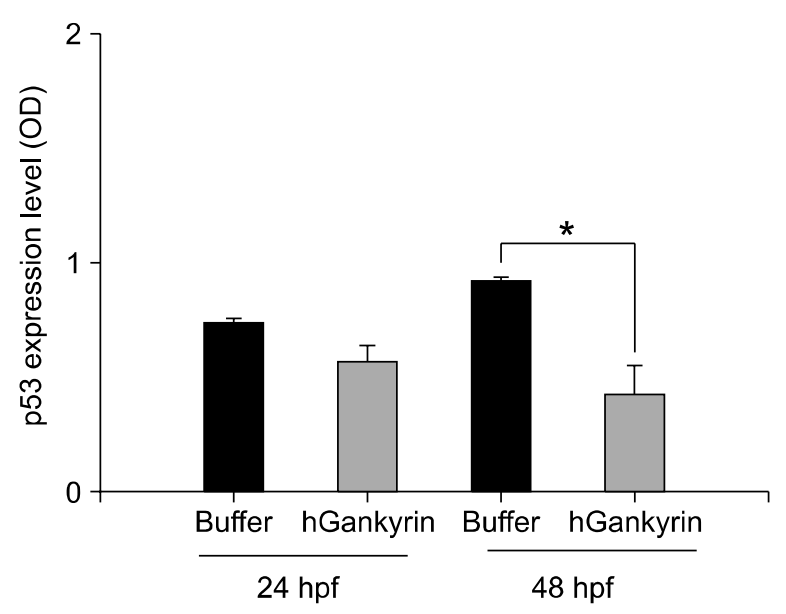

C

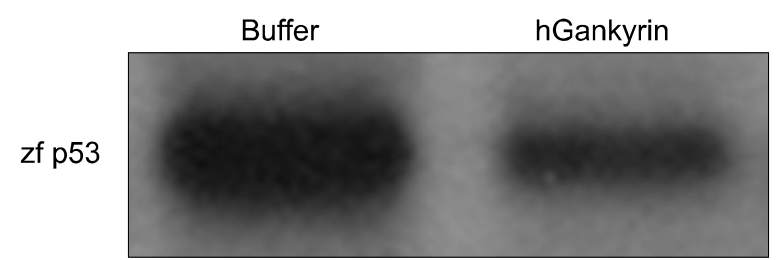

$28 S$

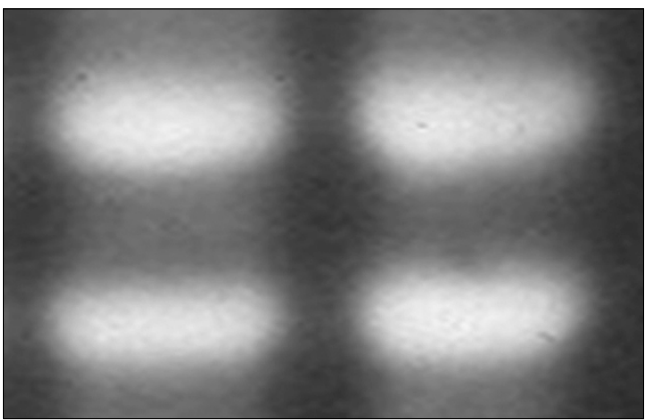

Figure 1. Alerted gene expression in gankyrin-injected zebrafish embryos. (A) Genes involving zf p53, zf p21 and Mdm2 were analyzed using single-embryo RT-PCR in embryos injected with pCS2+EGFP hgankyrin and buffer. Zf p53 and zf p21 expression was lower in embryos injected with hgankyrin compared to those injected with buffer as negative control. There was no difference in zf Mdm2 expression between hgankyrin injection and buffer, suggesting that hgankyrin regulates the expression of p53 but not Mdm2. (B) Zf p53 mRNA expression between injected with pCS2+EGFP hgankyrin and buffer was analyzed by RT-PCR. The results are determined as average ratios of the OD of zf p53 and to the OD of $\beta$-actin (average and standard errors of the three experiments, $P$ value $<0.05$ ). Above the results (Figure 1A), level of zf p53 only decreased in hgankyrin injection embryos. (C) Northern blot analysis of p53 and Mdm2 expression following hgankyrin overexpression in zebrafish. Total RNA were prepared at $24 \mathrm{hpf}$ of each lane and distributed by formaldehyde-agarose gel electrophoresis. $15 \mu \mathrm{g}$ of RNA were loaded in each lane and hybridized using p53 and Mdm2 probes. The p53 levels are down regulated in hgankyrin injected embryo at $48 \mathrm{hpf}$. The loading control for lanes serves 18S rRNA band photographed from the etiethidium bromide stained gel. 
cular pathways identified in zebrafish can be expected to be found in humans as well (Amatruda et al., 2002; Yang et al., 2004). In particular, the p53 and $\mathrm{Mdm} 2$ signal in zebrafish conserved in humans, hence, they are very useful in the identity of new drugs and genes affecting p53 signaling (Langheinrich et al., 2002). Thus, in the present study, we explored the relationship of gankyrin protein on the p53-mediated pathway during development of zebrafish.

\section{Results}

\section{Overexpression of hgankyrin decreases p53 level}

Previous study demonstrated that gankyrin reduced the levels of p53 mRNA and protein in in vitro study using human cell lines (Higashitsuji et al., 2005). In the present study, we wanted to know if gankyrin affect the p53 and Mdm2 levels in zebrafish. We injected hgankyrin or mock vector into zebrafish and performed RT-PCR and Nor-
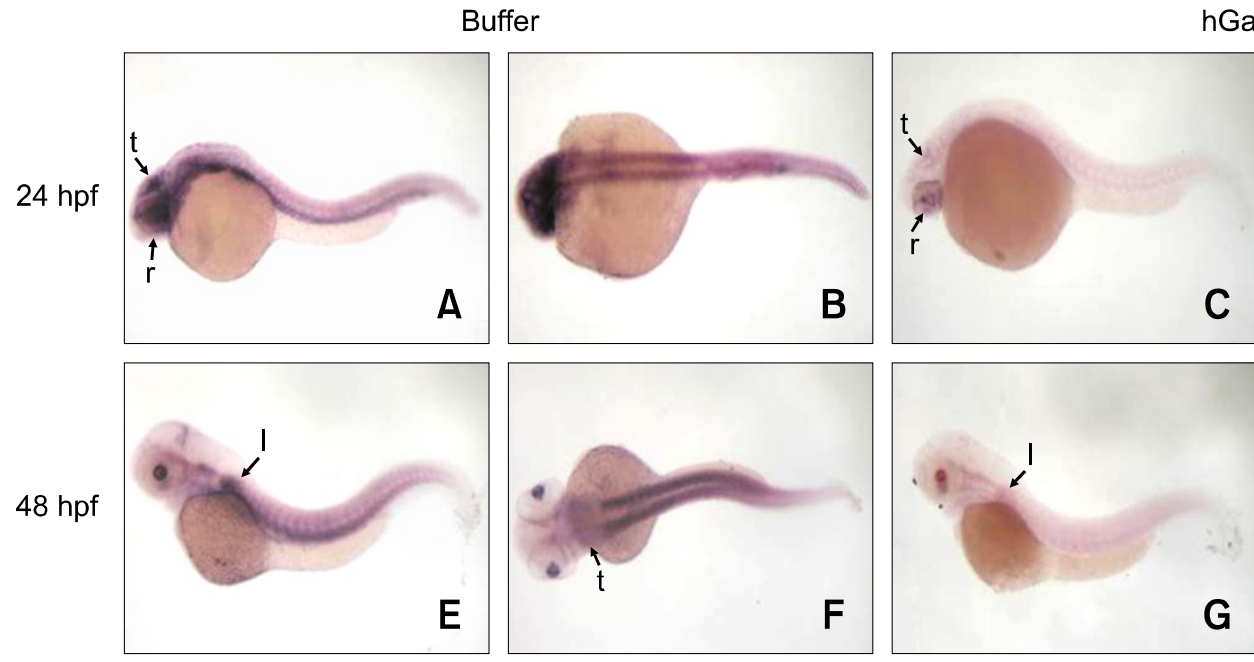

Gankyrin

Figure 2. Whole mount in situ hybridization using zf p53 probe at 24 and $48 \mathrm{hpf}$. Zf p53 expression was lower in embryos injected with hgankyrin (C, D,

$G, H)$ than that in embryos injected with buffer (A, B, E, F) as negative control. These results suggest that hgankyrin downregulates zf p53 mRNA levels. r, retina; $t$, tectum; l, lateral line primodium.
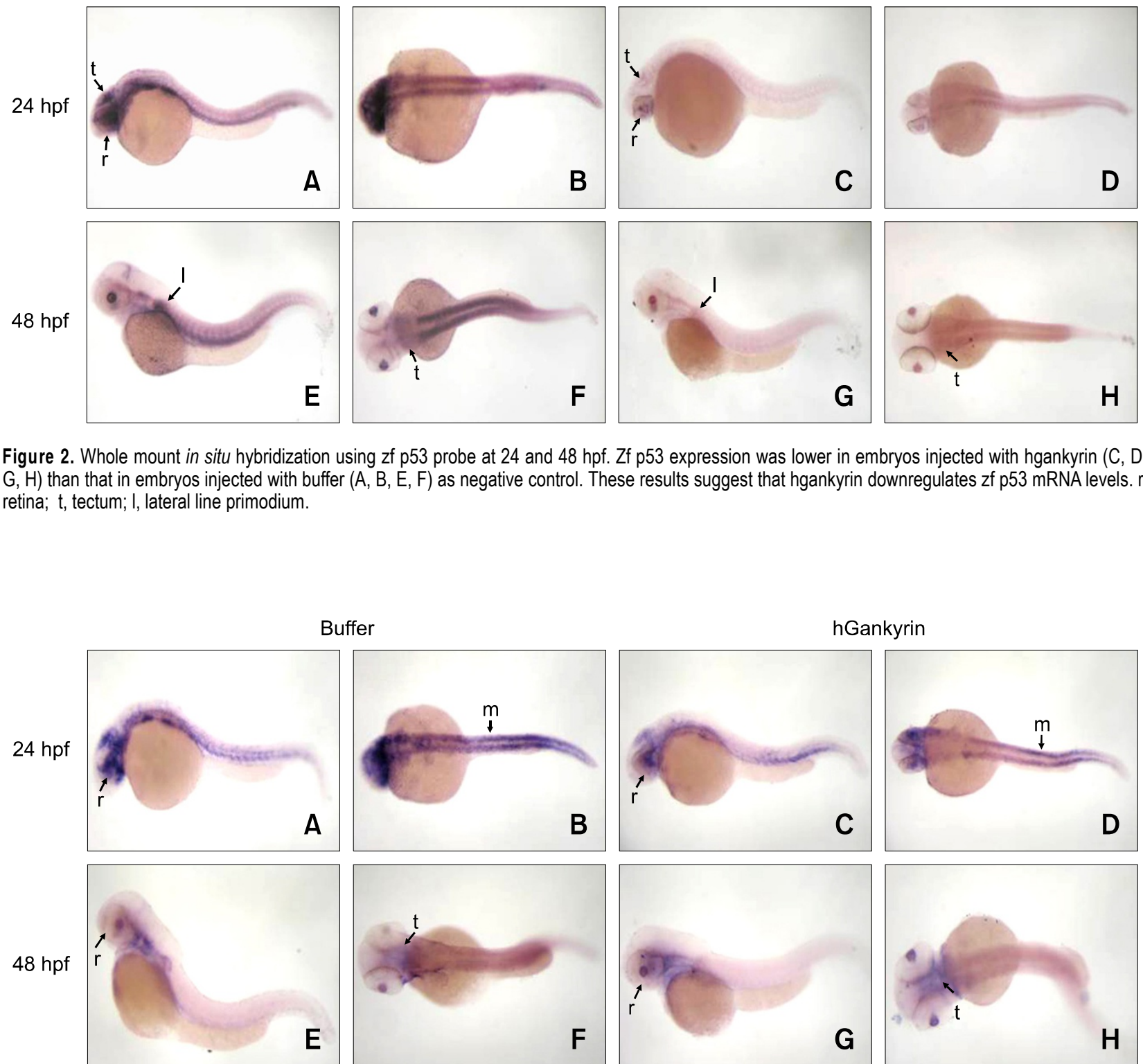

retina; t, tectum; l, lateralline primodium. 
thern blot analysis to determine p53, p21 and Mdm2 expression. In hgankyrin-injected embryos, zf p53 mRNA expression was slightly repressed at 24 hpf $(P<0.07)$ compared to that of bufferinjected embryos, but it was significantly repressed at 48 hpf $(P<0.02)$ compared to control (Figure $1 \mathrm{~A}, \mathrm{~B})$. We furthermore confirmed alteration of expression levels in zf p53 at 48 hpf with Northern blot (Figure 1C). Regarding to p21 mRNA expression, it was also decreased in hgankyrin-injected embryos compared to controls (Figure 1A). However, Mdm2 mRNA levels of hgankyrin-injected and buffer-injected cells exhibited little difference (Figure 1A). These results showed that gankyrin affected zf p53 and zf p21 mRNA levels but not zf Mdm2 levels.

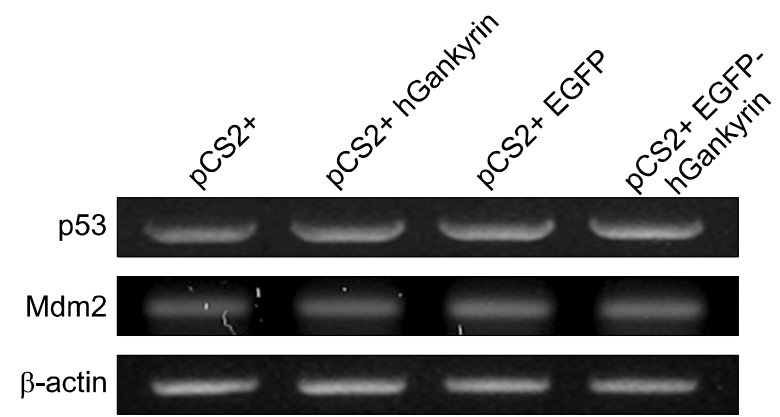

Figure 4. Effect of gankyrin mRNA level of p53 and Mdm2 in HEK 293 cells. HEK 293 cells were plated $60 \mathrm{~mm}^{2}$ dish and transfected with hGankyrin expression vector and Mock vector. mRNA level of p53 was not reduced by hgankyrin also mRNA level of Mdm2 remained almost unchanged our results.

\section{Expression pattern of $\mathrm{p} 53$ and Mdm2 by whole-mount in situ hybridization}

To compare the effects of gankyrin on the temporal and spatial expression of p53 and Mdm2, we performed whole-mount in situ hybridization. hGankyrin- and buffer-injected embryos had similar morphology but they differed at the p53 mRNA levels. The p53 mRNA expression was weaker in hgankyrin-injected embryos than in buffer-injected embryos at 24 (Figure 2A-D) and $48 \mathrm{hpf}$ (Figure 2, $\mathrm{E}-\mathrm{H})$, respectively. There was no significant difference in the mdm2 mRNA expression between the two groups (Figure 3). These results concur with the findings from RT-PCR, which stated that gankyrin was shown to affect only p53 mRNA levels and not Mdm2 mRNA levels.

\section{Effects of hgankyrin mRNA level of p53 in vitro}

To repeatedly confirm the relationship between gankyrin and p53 in our experiments, we transfected hgankyrin containing vectors and mock vector into HEK 293 cells. At 24 and 48 h posttransfection the cells were harvested and RT-PCR was performed. The p53 and Mdm2 mRNA levels were unchanged regardless of hgankyrin overexpression at both $24 \mathrm{~h}$ (Figure 4) and $48 \mathrm{~h}$ after transfection. Accordingly, these results showed that gankyrin did not affect p53 mRNA levels.

\section{Cell proliferation by trypan blue, MTT and BrdU incorporation assay}

In order to observe the effects of gankyrin on HEK 293 cell proliferation, we used trypan blue and
A

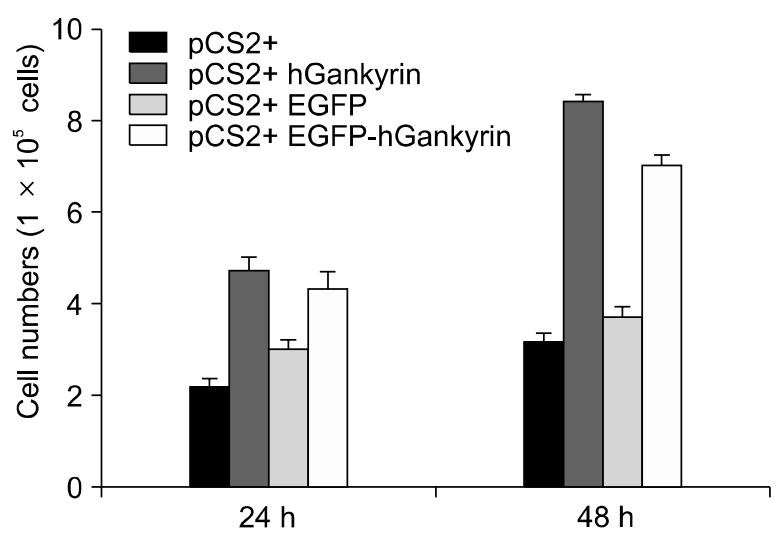

B

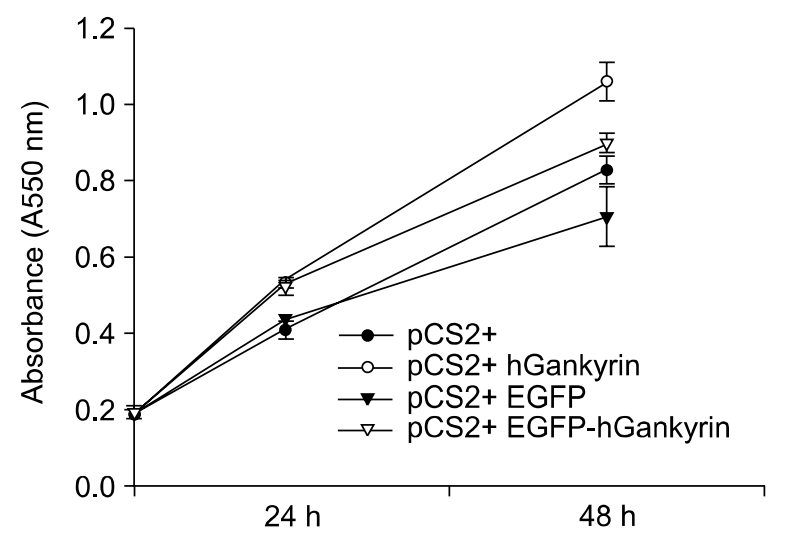

Figure 5. Effect of gankyrin on the proliferation of HEK 293 cells. (A) Effect of gankyrin on HEK 293 cell growth. The cell numbers were determined by trypan blue exclusion assay. hGankyrin-transfected HEK 293 cells experienced a higher rate of cell growth than the mock vector-transfected HEK 293 cells. (B) Growth curves of HEK 293 cells. Cells were seeded in 12 well plates and transfected with either hgankyrin expression vector or mock vector. Growth curves were determined by detecting reactions with MTT assay. Cell proliferation was greater in hgankyrin-transfected HEK 293 cells than in mock vector-transfected cells. 
MTT assays (Figure 5). The number of viable cells in the hgankyrin-transfected group increased approximately 1.5-3 fold compared to the mock-transfected group (Figure 5A) when cell growth was maintained for $48 \mathrm{~h}$. MTT assay was used to determine cell survival and proliferation rate. Similar to the results from the trypan blue assay, cell proliferation increased in hgankyrin-transfected cells, with a significant difference observed after $48 \mathrm{~h}$ post-transfection (Figure 5B). Thus we confirmed that gankyrin promotes cell growth activity in HEK 293 cells.

To investigate gankyrin's effect on cell proliferation in zebrafish embryos, we performed BrdU incorporation assays in embryos. BrdU positive cells were observed to be concentrated on the head and tail region in hgankyrin-injected embryos at 48 and $72 \mathrm{hpf}$, respectively (Figure 6A-L). The BrdU-positive cell numbers increase in hgankyrininjected embryos compared to buffer-injected embryos. Comparatively more BrdU-positive cells were observed in the neural crest (nc) (Figure 6A-D), ciliary marginal zone of eyes (cmz) (Figure 6A-B), midbrain-hindbrain boundary (mhb) (Figure 6C-D) (myotomes $(\mathrm{m})$ (Figure $6 \mathrm{E}-\mathrm{F})$, pectoral fin buds (pfb) (Figure 6G-H), and primodium (prim) (Figure $6 \mathrm{I}-\mathrm{L}$ ) at 48 and $72 \mathrm{hpf}$, respectively, but we found no difference in BrdU-positive cells in the trunk region. We speculate that gankyrin has a greater effect on zebrafish in the early stages of development.

\section{Discussion}

Gankyrin was initially cloned and characterized as the subunit of $26 \mathrm{~S}$ proteasome (Hori et al., 1998). It interacts with other proteins such as CDK4, Rb and $\mathrm{Mdm} 2$ which is a negative regulator of $\mathrm{p} 53$, to accelerate cell proliferation. p53 consists of 393 amino acids and, it regulated the cell cycle and apoptosis (Brown and Pagano, 1997). Decreased p53 activity will in turn lead to accelerated cell proliferation and repression of apoptosis (Lozano
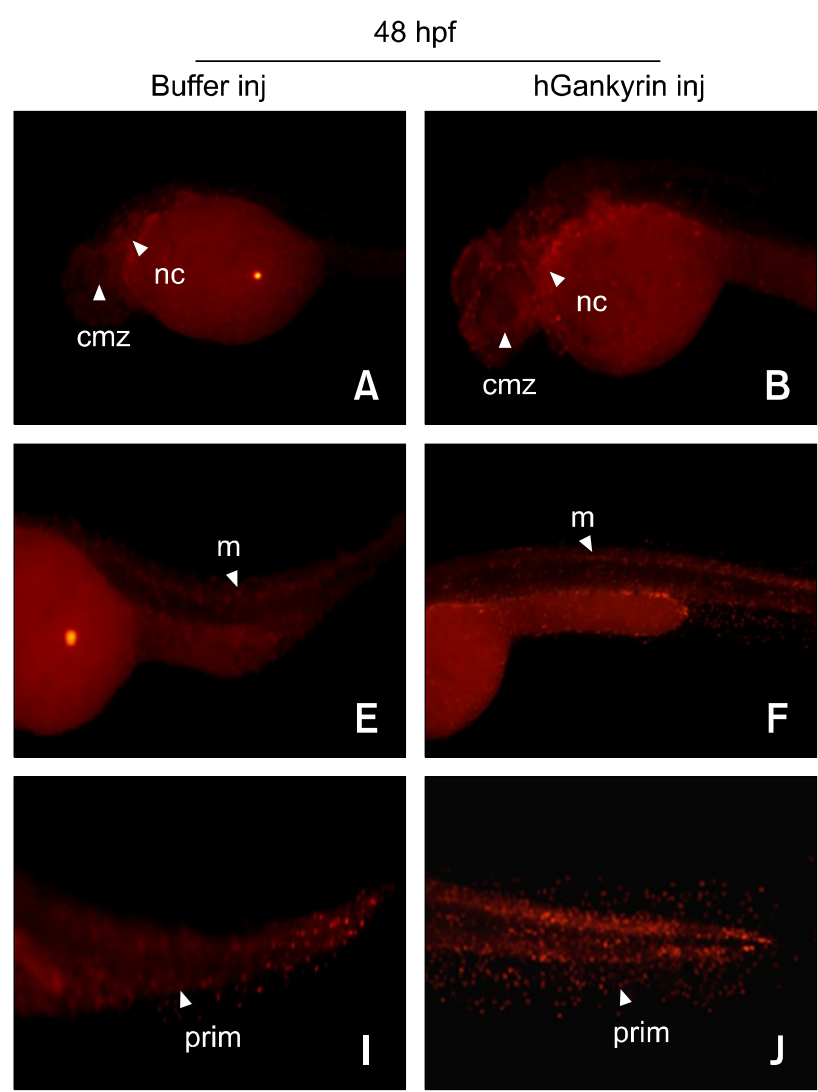

hGankyrin inj
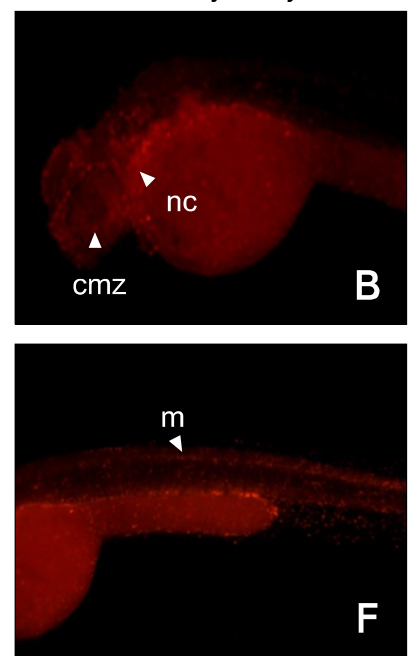

Figure 6. Gankyrin promotes cell proliferation. Embryos receiving pCS2+EGFP hgankyrin injections (B, J, D, H, L) exhibited more BrdU positive cells in their heads and tails than those receiving the control buffer injections (A, I, C, G, K). No differences in BrdU positive cells were observed in the trunk regions (E, F). nc, neural crest; pfb, pectoral fin buds; cmz, ciliary marginal zone of eyes; prim, primordium; mhb, midbrain-hindbrain boundary; m, myotomes. 
and Zambetti, 2005). p53 plays an important role in the suppression of tumor formation, and the loss of p53 function is related to tumorigenesis. Also p53 arrests the cell cycle or induces apoptosis in cells that experience DNA damage or other types of cellular stress (Nowak et al., 2005). In contract, Mdm2 is a protein targeted by $\mathrm{p} 53$ which binds to p53 through an $\alpha$-helix structure in the $\mathrm{N}$-terminal and stimulates the degradation of p53 via the action of a ubiquitin ligase ringer finger domain in the C-terminal (Daujat et al., 2001; Meek, 2004). In the present study, we studied correlation between gankyrin, and both p53 and Mdm2, in zebrafish. The level of p53 mRNA in vivo decreased when hgankyrin expression vectors were injected into embryos as opposed to when mock vector were injected in embryos, and the difference was distinct at $48 \mathrm{hpf}$ (Figure 1A). This report is the first to reveal evidence that effect of hgankyrin over-expression in zebrafish embryo includes the alteration of gene expression and increased proliferation. However, we could not detect the protein levels of zf p53 because cross-reactive antibody to zf p53 is not present. Our results are in contrary to the previous report by Higashitsuji et al (2005). showing that hgankyrin did not reduce the level of p53 mRNA but reduce p53 protein level in human cancer cell lines, suggesting that gankyrin affects the rate of synthesis and/or the stability of p53 protein. This discordance may be attributable to the difference in in vitro and in vivo microenvironment. Also, previous study by Langheinrich et al. (2002) showed that small sized p53 mRNA (1.7 $\mathrm{kb})$ was found in zebrafish embryos treated with camptothecin compared to that $(2.2 \mathrm{~kb})$ of control embryos. The reason of this mechanism remains unclear but they explained that the occurrence of the induced $1.7 \mathrm{~kb}$ transcript would be usage of the internal polyA signal site, which is present in zebrafish p53. Furthermore, they also suggest that p53 has been shown to autoregulate its own transcription in zebrafish, resulting in the regulation of p53 mRNA levels and p53 promoter activation, but this regulation mechanism is thought to be different with animal cells. These features might indicate a special zebrafish-specific step in regulating p53 activity (Langheinrich et al., 2002). Based on this study, we tried to investigate how gankyrin affects p53 and Mdm2 expressions during the development of zebrafish. We performed in situ hybridization following overexpression of hgankyrin in zebrafish embryos and examined the temporal and spatial expression alteration of p53 and Mdm2. Zf p53 and zf Mdm2 expression patterns were spatially specific. Zf p53 expression was observed predominantly in the head region, including in the retina and tectum, as opposed to low expression observed in the trunk region (Thisse et al., 2000; Cheng and $\mathrm{Wu}, 2002$ ) (Figure 2A, B, E, F), while zf Mdm2 expression was detected in prominent tissues, in particular the tectum, retina and myotomes (Thisse et al., 2000). Furthermore, zf p53 expression decreased at a higher rate in hgankyrin-injected zebrafish (Figure 2C, D, G, H) as opposed to buffer-injected zebrafish at both 24 and $48 \mathrm{hpf}$ (Figure 3A, B, E, F), but zf Mdm2 expression did not differ between hgankyrin-injected and buffer- injected at both 24 and $48 \mathrm{hpf}$ (Figure 3C, D, G, H). These results demonstrate that gankyrin regulates p53 expression but not Mdm2 during the development of zebrafish. This interesting observation is consistent with the previous report showing that gankyrin binds to Mdm2, enhancing ubiquitylation and degradation of p53 (Higashitsuji et al., 2005).

Next, in order to find out whether hgankyrin overexpression leads to cell proliferation, cell viability and cell proliferation assay were performed. Trypan blue assay revealed that the viable cell numbers increased in hgankyrin expressing vector as compared with mock vector transfected cells. Significant difference in cell viability between the two groups was observed at $48 \mathrm{~h}$ incubation after transfection (Figure 5A). To evaluate the cell proliferation rate, the growth pattern affected by hgankyrin transfection was determined by using MTT assay. Acceleration in cell growth was observed in cells transfected with hgankyrin, compared to cells transfected with mock vector (Figure 5B). This result suggests that hgankyrin overexpression is related to cell proliferation.

For the investigation of cell proliferation by hgankyrin overexpression in vivo, BrdU immunostaining was done in hgankyrin injected zebrafish embryo. We found that BrdU positive cells had a greater presence in hgankyrin-injected embryos than the buffer-injected embryos, in particular, the head and tail regions (Figure 6).

In detail, BrdU positive cells were particularly concentrated in the neural crest, pectoral fin buds, ciliary marginal zone of eyes, midbrain-hindbrain boundary, primodium and myotomes (Figure 6). BrdU positive cells were observed after $24 \mathrm{hpf}$, however, difference between hgankyrin injected embryo and buffer injected embryo was distinctly observed at 48 and $72 \mathrm{hpf}$. Therefore we speculate that gankyrin has a greater effect at later stages during development.

Consequently, there was a greater decrease in p53 mRNA levels in hgankyrin injected embryo compared to the mock vector injected embryos. With the exception of p53 mRNA levels, other 
results were similar in vitro and in vivo suggesting that zebrafish can be a useful tool in the understanding of cellular regulating mechanisms related to gankyrin. In the future, further investigation on the effects and functions of gankyrin in development and tumorigenesis by looking at zebrafish gankyrin knock-down with antisense morpholino will be necessary.

\section{Methods}

\section{Fish care}

Zebrafish were maintained at $28.5^{\circ} \mathrm{C}$ with alternating photoperiods of $14 \mathrm{~h}$ and darkperiods of $10 \mathrm{~h}$ in our aquarium facility. Egg collection and developmental stage classification was done as previously described (Westerfield, 1995).

\section{Plasmid construction}

The cDNAs of full-length human gankyrin (hgankyrin) were amplified by RT-PCR using total RNA extracted from human hepatoma cell line, HepG2. The primer sequences used were 5'-ACTGGCGTAGCCGGAGCCGGC-3' (sense primer, nucleotides nt 28-48 in GenBank Accession No. D83197 for 22nt position numbering) and 5'-GGACACTGGGGACAACAACAC-3' (antisense primer, nt 811831). The amplified fragments were inserted into pGEMT-easy vector (Promega, Madison, WI), designated pGEMT-easy hgankyrin. After digestion of pGEM-T-easy hgankyrin with appropriate restriction enzymes, isolated cDNA of hgankyrin was cloned into the pEGFP-C2 (Clontech, Palo Alto, CA) and pCS2+ vector (Clontech). The following hgankyrin constructs were generated: pCS2+hgankyrin and pEGFP- hgankyrin. After that the pEGFP-hgankyrin is digested with the appropriate restriction enzymes and ligated into the multiple cloning region of a linearized pCS2+ and then the construct pCS2+EFGP-hgankyrin was established.

\section{In vitro transcription and microinjection of zebrafish embryos}

Human gankyrin expression vectors pCS2+hgankyrin and pCS2+EFGP-hgankyrin were linearized by using Not I and the pCS2+EGFP vector was linearized by using Nco I, respectively. These vectors were purified with phenol: chloroform and precipitated with ethanol. The linearized plasmid DNA was transcribed in vitro using a SP6 mMESSAGE Kit (Ambion, Molecular Biochemicals, Mannheim, Germany). This in vitro transcribed RNA was mixed with $0.5 \%$ phenol red at a $1: 1$ ratio and then was injected through intact chorion into the yolk at the base of the blastomeres of one- to four-cell stage embryos at a concentration of $200 \mathrm{ng} / \mu \mathrm{l}(100-200 \mathrm{pg} / \mathrm{embryo})$. The controls for each experiment were $0.5 \%$ phenol red-injected (buffer), pCS2+ EGFP-injected (100-200 pg/embryo) and uninjected (wild) embryos.

\section{RNA isolation and RT-PCR}

Total RNA was isolated from hgankyrin- and bufferinjected embryos at 24 and $48 \mathrm{~h}$ post fertilization (hpf) and from transfected cells with hgankyrin constructs using Trizol reagent (Invitrogene, Grand Island, NY). One $\mu \mathrm{g}$ of total RNA was used for reverse transcription with a random primer. Zebrafish p53 (zf p53), p21 (zf p21) and Mdm2 (zf $M d m 2$ ) cDNA were amplified using the primers as previously described by Liu et al. (2003). The sequences of used primers are as follows: for $z f$ p53, sense 5'TGTCAGCTGGCAAAAACTTG-3', antisense 5'-ACAAAGGTCCCAGTGGAGTG-3'; for zf p21 WAF/CIP1 sense 5'-TGAGAACTTACTGGCAGCTTCA-3', antisense 5'-AGCTGCATTCGTCTCGTAGC-3'; for zf Mdm2, sense 5;-CAGCAAGGTTGACAACGAGA-3', antisense 5'-CGAAGGTTGTGTTGGGAGTT-3', zf $\beta$-actin sequences were constructed according to GenBank Accession No. AF057040, sense 5'-CCCCTTGTTCACAATAACCT-3, antisense 5'TCTCTGTTGGCTTTGGGATTCA-3'. The sequences of PCR primers of human $\mathrm{p} 53, \mathrm{Mdm} 2$ and $\beta$-actin are as follows: P53 (GenBank Accession No. AF307851) sense 5'-AGATCTACCATGGAGGAGCCGCAGTCAGA-3', antisens 5'GAATTCTCAGTCTGAGTCAGGCCCTT-3'; Mdm2 (GenBank Accession No. BC013136) sense 5'-GGTTGACTCAGCTTTTCCTCTTG-3', antisense 5'-GGAAAATGCATGGTTTAAATAGCC-3'; $\beta$-actin (GenBank Accession No. BC002409) sense 5'-AGGCCAACCGCGAGAAGATGACC3', antisens 5'-GAAGTCCAGGGCGACGTAGCAC-3'. The PCR products were analyzed on $1.5 \%$ agarose gels. The $\mathrm{OD}$ of the bands on the gel was determined using TINA 2.09G software.

\section{Northern blot analysis}

Total RNA was prepared from hgankyrin- and bufferinjected embryos at 24 and $48 \mathrm{hpf}$ using Trizol (Invitrogen, Carlsbad, CA). RNA of $15 \mu \mathrm{g}$ per lane were separated in $1.2 \%$ agarose-formaldehyde gels and electrotransferred onto positively charged nylon membranes (Roche Diagnostics, Mannheim, Germany). RNA blotters were crosslinked using UV irradiation and probed with ${ }^{32} \mathrm{P}$-labeled $z f$ p53 and zf Mdm2 cDNA. The blotters were incubated overnight in hybridization buffer (1\% BSA, $7 \%$ SDS, $0.5 \mathrm{M}$ $\mathrm{NaH}_{2} \mathrm{PO}_{4}, 1 \mathrm{mM}$ EDTA at $65^{\circ} \mathrm{C}$. The blotters were washed twice in $2 \times$ SSC, $0.1 \%$ SDS for $5 \mathrm{~min}$ at $37^{\circ} \mathrm{C}$, two more times in $1 \times$ SSC, $0.1 \%$ SDS for 30 min at $65^{\circ} \mathrm{C}$ and once more in $0.2 \times \mathrm{SSC}$ for $5 \mathrm{~min}$ at $37^{\circ} \mathrm{C}$. Following this blotters were then exposed to AGFA X-lay blue film (AGFA Gevaert, N.V.) with intensifying screens.

\section{Whole mount in situ hybridization}

Zf p53 and zf Mdm2 were digested with Hind III and BamH $I$, respectively. All linearized plasmids were transcribed in vitro using SP6 polymerase using digoxigenin-labeld UTP (Roche). Whole mount in situ hybridization was performed as described previously (Westerfield, 1995). Images of zebrafish embryos were recorded using a Leica MZ FL III microscope (Leica Mikrosysteme, Bensheim, Germany) and a Canon Powershot S70 Digital Camera (Canon USA Inc., Lake Success, NY) at $50 \times$ magnification. 


\section{BrdU incorporation assay}

To detect cell proliferation in zebrafish, we performed BrdU (5'-bromodeoxyuridine; Sigma, MO) incorporation. To incorporate BrdU (Sigma) into DNA, we manually dechorinated zebrafish embryos and soaked them in chilled embryo medium with $10 \mathrm{mM}$ BrdU (Sigma) and 15\% DMSO (Amresco, OH) in solution. Following this step, embryos were fixed with $4 \%$ paraformaldehyde. After removing paraformaldehyde, the embryos were put in fresh methanol at $-20^{\circ} \mathrm{C}$ for at least $1 \mathrm{~h}$ and then rehydrated using $75 \%, 50 \%$, and $25 \%$ methanol in series. Embryos were incubated in $2 \mathrm{~N} \mathrm{HCl}$ for $1 \mathrm{~h}$ and treated with blocking solution (PBS-D-Tw[PBS, 1\% DMSO, 0.1\% Tween-20], 1\% BSA, $2 \%$ normal goat serum) for $10 \mathrm{~min}$. Embryos were incubated with polyclonal anti-BrdU (1:100, Dako, Glostrup, Denmark) overnight at $4^{\circ} \mathrm{C}$ and incubated with secondary Cy3-conjugated anti mouse (Jackson ImmunoResearch Laboratories) for 3-4 h using standard conditions. Fluorescence was viewed using a widefield fluorescence microscopy on a ZEISS Lumar.V12 stereomicroscope (Zeiss, Jena, Germany) with filter sets designed for EGFP or Cy3. Digital images were taken using a CoolSnap color camera (Zeiss) and Axiovision software.

\section{Cell culture and transfection}

Human embryonic kidney (HEK) 293 cells (American Type Culture Collection, Manassas, VA) were cultured in DMEM (Invitrogen) supplemented with 10\% FBS (Invitrogen), 50 $\mathrm{U} / \mathrm{ml}$ penicillin, and $50 \mathrm{~g} / \mathrm{ml}$ streptomycin (Invitrogen). For transfection, $1 \times 10^{6}$ cells were seeded $60 \mathrm{~mm}^{2}$ dish and transfected with an each $1 \mu \mathrm{g}$ hgankyrin expression vector and mock vector using Lipofectamine (Invitrogen) reagent.

\section{Measurement of cell growth by trypan blue staining and MTT assay}

To measure the proliferation of gankyrin, HEK 293 cells were plated on $1 \times 10^{5}$ cells/wells ( 6 well plate) and transfected each $0.5 \mu \mathrm{g}$ hgankyrin expression vector and mock vector using Lipofectamine (Invitrogen) reagent. The samples in the six-well plates were collected by trypsinization and the viable cell number was measured using trypan blue staining using light microscopy. Also $2 \times 10^{4}$ cells/wells (12 well plate) were incubated until $70-80 \%$ confluent, and then transfected with $0.2 \mu \mathrm{g}$ hgankyrin expression vector and mock vector using Lipofectamine reagent.

24 and $48 \mathrm{~h}$ post-transfection the cells had their media replaced with $400 \mu \mathrm{l}$ MTT solution (Sigma; $0.5 \mathrm{mg} / \mathrm{ml}$ ) per well, and were incubated for $2 \mathrm{~h}$ at $37^{\circ} \mathrm{C}$ in a humid $5 \%$ $\mathrm{CO}_{2}$ cell incubator. Then $100 \mu \mathrm{l}$ DMSO and $100 \mu \mathrm{l}$ of each sample were transferred to 96 -well plates and $O D$ analysis was performed at a wavelength of $550 \mathrm{~nm}$ using Microplate (Elisa) reader (Molecular Devices, Sunnyvale, CA).

\section{Statistical analysis}

To analyze the statistical differences in expression of zf p53 between hgankyrin-injected embryos and bufferinjected embryos, a one-way analysis of variance (ANOVA) was used. Significance was defined as $P<0.05$.

\section{Acknowledgements}

This work was supported by FG08-12-05 of the 21C Frontier Functional Human Genome Project from the Ministry of Science and Technology in Korea. We are grateful to Dr. Cheol-Hee Kim (Chungnam National University) and Hyun-Taek Kim (Chungnam National University) for setting up the Zebrafish facilities.

\section{References}

Amatruda JF, Shepard JL, Stern HM, Zon LI. Zebrafish as a cancer model system. Cancer Cell 2002;1:229-31

Brown JP, Pagano M. Mechanism of p53 degradation. Biochim Biophys Acta 1997;1332:01-6

Cheng R, Wu JL. Gene expression of insulin-like growth factor-I receptor and p53 suppressor during zebrafish (Danio rerio) embryogenesis. Mar Biotechnol (NY) 2002;4:226-35

Daujat S, Neel H, Piette J. MDM2: life without p53. Trends Genet 2001;17:459-64

Higashitsuji H, Itoh K, Nagao T, Dawson S, Nonoguchi K, Kido T, Mayer RJ, Arii S, Fujita J. Reduced stability of retinoblastoma protein by gankyrin, an oncogenic ankyrinrepeat protein overexpressed in hepatomas. Nat Med 2000;6:96-9

Higashitsuji $\mathrm{H}$, Higashitsuji $\mathrm{H}$, Itoh $\mathrm{K}$, Sakurai T, Nagao T, Sumitomo Y, Masuda T, Dawson S, Shimada Y, Mayer RJ, Fujita J. The oncoprotein gankyrin binds to MDM2/HDM2, enhancing ubiquitylation and degradation of p53. Cancer Cell 2005;8:75-87

Hori T, Kato S, Saeki M, DeMartino GN, Slaughter CA, Takeuchi J, Toh-e A, Tanaka K. cDNA cloning and functional analysis of p28 (Nas6p) and p40.5 (Nas7p), two novel regulatory subunits of the $26 \mathrm{~S}$ proteasome. Gene 1998; 216:113-22

Iwai A, Marusawa H, Kiuchi T, Higashitsuji H, Tanaka K, Fujita $\mathrm{J}$, Chiba T. Role of a novel oncogenic protein, gankyrin, in hepatocyte proliferation. J Gastroenterol 2003;38:751-8

Krzywda S, Brzozowski AM, Higashitsuji H, Fujita J, Welchman R, Dawson S, Mayer RJ, Wilkinson AJ. The crystal structure of gankyrin, an oncoprotein found in complexes with cyclin-dependent kinase 4 , a 19 S proteasomal ATPase regulator, and the tumor suppressors $\mathrm{Rb}$ and $\mathrm{p} 53$. J Biol Chem 2004;279:1541-5

Langheinrich U, Hennen E, Stott G, Vacun G. Zebrafish as a model organism for the identification and characterization of drugs and genes affecting p53 signaling. Curr Biol 2002;12:2023-8

Li J, sai MD. Novel insights into the INK4-CDK4/6-Rb pathway: counter action of gankyrin against INK4 proteins regulates the CDK4-mediated phosphorylation of $\mathrm{Rb}$. Biochemistry 2002;41:3977-83

Liu TX, Howlett NG, Deng M, Langenau DM, Hsu K, Rhodes J, Kanki JP, D'Andrea AD, Look AT. Knockdown of zebrafish 
Fancd2 causes developmental abnormalities via p53dependent apoptosis. Dev Cell 2003;5:903-14

Lozano G, Zambetti GP. Gankyrin: an intriguing name for a novel regulator of p53 and RB. Cancer Cell 2005;8:3-4

Meek DW. The p53 response to DNA damage. DNA Repair (Amst) 2004;3:1049-56

Nowak M, Koster C, Hammerschmidt M. Perp is required for tissue-specific cell survival during zebrafish development. Cell Death Differ 2005;12:52-64

Park TJ, Kim HS, Byun KH, Jang JJ, Lee YS, Lim IK. Sequential changes in hepatocarcinogenesis induced by diethylnitrosamine plus thioacetamide in Fischer 344 rats: induction of gankyrin expression in liver fibrosis, $p R B$ degradation in cirrhosis, and methylation of $p 16$ (INK4A) exon 1 in hepatocellular carcinoma. Mol Carcinog 2001;30: $138-50$
Qin JM, Yan HX, Wan XW, Liu SQ, Zeng JZ, Cao HF, Wu MC, Wang HY. Effects of signal regulatory proteinalpha1 on proliferation of hepatocellular carcinoma: a preliminary study. Hepatobiliary Pancreat Dis Int 2005;4:244-8

Thisse C, Neel H, Thisse B, Daujat S, Piette J. The Mdm2 gene of zebrafish (Danio rerio): preferential expression during development of neural and muscular tissues, and absence of tumor formation after overexpression of its CDNA during early embryogenesis. Differentiation 2000;66:61-70

Westerfield M. The zebrafish Book : A Guide for Laborator use of Zebrafish (Danio rerio). University of Oregon Press, Eugene, OR 1995

Yang HW, Kutok JL, Lee NH, Piao HY, Fletcher CD, Kanki JP, Look AT. Targeted expression of human MYCN selectively causes pancreatic neuroendocrine tumors in transgenic zebrafish. Cancer Res 2004;64:7256-62 